\title{
A transformation, augmentation, substitution service (TASS) to meet the needs and preferences of the individual learner.
}

\author{
Voula Gkatzidou \\ University of Teesside \\ s.gkatzidou@tees.ac.uk
}

\author{
Elaine Pearson \\ University of Teesside \\ e.pearson@tees.ac.uk
}

\begin{abstract}
The accessibility community is responding to the challenges of Web 2.0 by adopting a more holistic approach to the provision of inclusive e-learning. It is based on the premise that an equivalent learning outcome, rather than a universally accessible single resource, meets the needs of individual learners more appropriately. This research suggests a solution to the creation of learning resources, which is based on the principle that learning content can be generated from adaptable aggregations of learning objects and media components using proven learning patterns. A transformation, augmentation, substitution service (TASS) encompasses methods to retrieve a learning object upon request by a user, adapt it and deliver it to the learning in the form in which it is required.
\end{abstract}

\section{Introduction}

Recent work on Web 2.0 has espoused a holistic approach to Web accessibility for e-learning which is based on accessible learning outcomes, rather than accessible resources, and treats the student not as someone who is disabled but as someone with alternative learning preferences [7]. The newly emerged term 'Accessibility 2.0' aims to provide a means of articulating a shift in the approaches of addressing accessibility, by acknowledging that 'one size fits all' approaches can be impractical, and encouraging creativity in providing multiple, aggregated routes that together help as many as possible to achieve the same goal. Inclusive learning environments should treat the student not as someone who is disabled, but as someone with alternative learning preferences and place the emphasis not on the outcomes for the individual learners, but on the objective qualities of the resource [8].

An approach which complements the holistic model is the development of a metadata framework, which can label aspects of a resource related to its accessibility, allowing for the selection of variants of a resource which are best suited for the individual user at the time and in a given context. The needs and preferences of a user may arise from the context or environment the user is in, the tools available (e.g. mobile devices, assistive technologies such as Braille devices, voice recognition systems or alternative keyboards etc.), their background or a disability (physical, cognitive or sensory). Taking accessibility in its widest sense and accepting user preferences, this research focuses on extending the possibilities of learning object adaptation by proposing a transformation, augmentation, substitution service (TASS), which by using a variant of IMS AccessForAll [6] works on available metadata and user profiles to generate alternative, equivalent learning experiences relating to a users' declared needs, preference and learning styles.

\section{Background Research}

According to the AccessForAll metadata overview, in order to achieve an accessible relationship between the resource and the user, a description of a user's control, display and content needs and preferences is matched with a description of the components of the learning object [9]. This may involve the substitution, augmentation or transformation of components of the resource, such as changes in sensory modality.

We propose a transformation, augmentation and substitution service (TASS), which can be viewed as a special instance of an AccessForAll service. Our work to date has focused on applying the TASS to learning objects. A basic resource needs to be created from existing, or newly authored components and the appropriate adaptations (transformations, augmentations or substitutions) need to be identified. Transformation may occur where text is rendered visually, as characters, or a sign language, or aurally, perhaps by a screen reader, or transformed into a tactile form as Braille or simply changed in colour, size and other display features. Augmentation involves the optional addition of a feature to a primary resource, for instance a textual caption could be added to a video when required by a user with a hearing impairment or in a noisy environment. Substitution might occur when 
a user requires a vision-free access to the resource.

A number of projects have been carried out in the direction of delivering personalised learning content on the basis of students' learning preferences [2]; [11]. These projects offer an insight into the issues of delivering personalised learning content. Our work combines accessibility issues with methodologies to automatically produce reusable and pedagogical learning objects that could be adapted to suit the diverse needs of learners, devices and environment.

\section{TASS Accessibility Service}

TASS is endowed with methods to retrieve a learning object upon request by a user, unpack and disaggregate it, execute the adaptation process, reaggregate its components to obtain a new learning object, and deliver it to the user [5]. Each of these activities is associated to a software component, which is deployed in a software architecture (Figure 1).

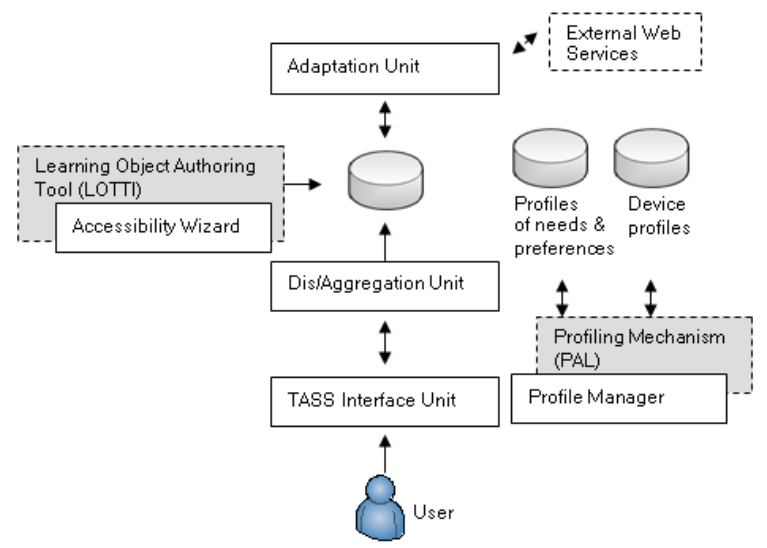

Figure 1. TASS System Architecture

Specifically, TASS comprises a number of software components implementing the functional activities: a TASS Interface Unit, a Profile Manager, an Accessibility Wizard, a Dis/Aggregation Unit and an Adaptation Unit.

When the user requests a learning object, the system authenticates to the TASS Interface Unit, which interrogates the Profile Manager, and retrieves the user's profile. The Interface Unit passes the request to the Dis/Aggregation Unit, which fetches the requested learning object from the learning object repository, unpackages it and defines a correspondent adaptation strategy, based on the learner's profile and the available alternative learning object components. The strategy, along with the media that need conversion is forwarded to the Adaptation Unit, which performs the necessary adaptations as specified by the strategy and upon their completion, returns the learning object to the user, via the TASS Interface Unit. The Adaptation Unit might also engage a set of Web services designed to accomplish specific adaptation processes, such as media file or text to speech conversions.

The two main building blocks of TASS are the Accessibility Wizard and the Profile Manager, which are both integrated into two stand-alone applications, the learning object authoring tool (LOTTI) and the Profile for Adaptable Learning (PAL) tool. The Accessibility Wizard component within LOTTI allows the author to design and develop learning objects, identify alternative component resources, and assign the appropriate metadata to it. The Profile Manager component within PAL allows users to create and manage their Profile of Needs and Preferences (PNP), based on the use of the IMS metadata specifications. The work to date has focused in the development of prototypes of these components, and their functionality is described in the following section.

\section{Authoring adaptable learning objects}

The Learning Object Tutor Tools Interface (LOTTI) is an authoring tool for academics to aggregate and publish learning objects. With the aid of learning design patterns, the tool will allow the designer to create and appropriately label retrievable, re-usable and adaptable learning objects [4]. Creating accessible and universal learning objects is a difficult and lengthy task, partially supported by complicated ad-hoc tools [3], therefore LOTTI aims to provide an easy way for an academic with limited programming experience, to create learning objects that could be retrieved, shared and re-purposed over the Internet.

LOTTI adopts the Generative Learning Object (GLOs) method of learning object creation, by separating the design process into two steps, the first one consists of building a learning object template which encloses the deep general structure of the object while the second one involves adding specific learning content to the template. [1]. Once a template has been created, authors can add different subject specific content to produce Learning Objects.

The Accessibility Wizard is triggered when the learning designer imports a learning object component; it prompts the designer to assign an alternative resource, outlines the limitations of the file type to the designer and suggests predefined alternatives to these types of content to the user.

\section{Profiling learners}

The Profile for Adaptable Learning (PAL) is a tool which provides an easy way to create a learner's 
profile, where the Profile Manager allows learners to create, edit and modify their preferences regarding the appearance, layout and selection of content. Users can also customize the selection and presentation of content based on requests for alternative formats, such as captions, audio descriptions, or sign language.

PAL responds to the need for a method to provide a detailed learning profile, by implementing IMS ACCLIP in order to model the learner's access requirements. ACCLIP describes the user in terms of accessibility needs and enables the description of user preferences (visual, aural or device), which can be usefully exploited for tailoring learning content.

In order to make the learner profiling process as easy and straightforward as possible for the user, PAL summarises the general preferences into six adaptability statements. Each adaptability statement presents the user with a number of additional preference questions, related to the basic chosen statement. Once the learner profile has been detailed during the first access to the PAL system, it is typically stored in a database, to facilitate future interactions. Each time a request is made by the user, the Profile Manager passes the user profile to the adaptation unit, which triggers and orchestrates an adaptation process, specifically designed for the needs and preference of the specific user.

\section{Conclusions and Further Work}

This paper suggests an approach for designers of learning objects to respond to the challenge of providing truly learner centred, adaptable learning resources.

While TASS on its own is a useful tool for the creation and retrieval of adaptable learning objects, we have begun to consider its potential as part of a complete learning environment. The concept of TASS can be extended to deal with the features of a learning environment as well as the content. It is possible to realize a development framework for an open source Adaptable Personal Learning Environment (APLE) [10] that is responsive to an individual learner's needs or preferences, and to the environment in which they are working.

\section{References}

[1] Boyle, T. (2003). Design principles for authoring dynamic, reusable learning objects. Australian Journal of Educational Technology, 19(1), pp. 46-58.

[2] Di Iorio, A., Feliziani, A., Mirri, S., Salomoni, P., \& Vitali, F. (2006). Automatically Producing Accessible Learning Objects. Educational Technology \& Society, 9(4), pp. 3-16.

[3] Dolog, P., Henze, N., Nejdl, W., \& Sintek, M. (2004). Personalization in Distributed eLearning Environments. Paper presented at the International World Wide Web Conference, New York, USA

[4] Gkatzidou, S., \& Pearson, E. (2007). Vodcasting: A case study in adaptability to meet learners' needs and preferences. Paper presented at the Australasian Society for Computers in Learning in Tertiary Education ASCILITE Conference. Singapore.

[5] Green, S., Jones, R., Pearson, E., \& Gkatzidou, S. (2006). Accessibility and adaptability of learning objects: responding to metadata, learning patterns and profiles of needs and preferences. ALT-J Research in Learning Technology, 14(1), pp. 117-129

[6] IMS. (2004). IMS Global learning/ Dublin Core AccessForAll project. Available online:

http://www.imsglobal.org/accessibility. Retrieved: 29/01/09

[7] Kelly, B., Nevile, L., Draffan, E., \& Fanou, S. (2008). One World, One Web...But Great Diversity. Paper presented at the International World Wide Web Conference. Beijing, China.

[8] Nevile, L., \& Treviranus, J. (2006). Interoperability for Individual Learner Centered Accessibility for Web-based Educational Systems. Journal of Educational Technology \& Society. 9(4)

[9] Nevile, L., Cooper, M., \& Heath, A. (2005). Learnercentred Accessibility for Interoperable Web-based Educational Systems. Paper presented at the International World Wide Web Conference. Chiba, Japan.

[10] Pearson, E., Green, S., \& Gkatzidou, S. (2008). Enabling Learning For all Through Adaptable Personal Learning Environments. Paper presented at the Australasian Society for Computers in Learning in Tertiary Education Conference (ASCILITE). Melbourne, Australia.

[11] TILE (2006). The Inclusive Learning Exchange. Available online: http://barrierfree.ca/tile/index.htm. Retrieved: 29/01/09 